\title{
NEW COLLABORATIVE ORGANIZATIONS AND THEIR RESEARCH NEEDS
}

\author{
Luis M. Camarinha-Matos \\ New University of Lisbon,PORTUGAL, cam@ uninova.pt
}

\begin{abstract}
This paper summarizes the interim findings of the THINKcreative project in terms of the required research agenda for new collaborative, networked organizations. A number of mechanisms including a Delphi survey, several focused workshops, panels, and regional workshops, were used to collect and synthesize the opinions and recommendations from a large number of industry and academic visionaries. The results of these initiatives are presented in terms of a collection of recommendations.
\end{abstract}

\section{INTRODUCTION}

The combination of two factors - the possibilities offered by the fast progress on computer networks and communication technologies, and the markets turbulence and globalization - are inducing new forms of collaboration. In addition to the traditional concept of supply chain and the first models of virtual enterprise / virtual organization, new organizational forms are emerging. Furthermore the pace of change or evolution in such collaborative organizations is very high, directing the focus of attention to the dynamic / evolutionary aspects of networks. Examples of this trend include: rapid formation of teams for emergency situations / rescue teams (characterized by a scenario with multiple leaders, lack of information and urgency in making decisions), collaborative entertainment networks (an emerging culture with teenagers), collaborative remote diagnosis, collaborative problem solving (e.g. collaborative data mining), virtual institutes (education), elderly care virtual communities, etc. Understanding the structure and associated behavior of these collaborative forms as well as the required infrastructures, support tools, regulations and operating principles, economic models, and support institutions, are major research challenges.

Motivated by these challenges, the THINKcreative project was launched as a "thinking network of experts on emerging smart organizations". The general mission of this project is to identify and characterize emerging collaborative organizational forms, their required infrastructures, modeling formalisms, and software tools, as well as their associated socio-organizational, ethical and moral changes, within the time frame of 5,10 , and 15 years.

This work is coordinated by a core team of 11 experts covering a wide spectrum of complementary expertise, including among others: VOs and collaborative 
systems, interoperation, knowledge and information management, software engineering, business process modeling, economy and management, social and ethical issues, and electronic business. Through a number of activities - focused workshops, regional workshops, discussion forum and panels, and a Delphi survey, a large number of other international experts and European industry leaders and visionaries were involved in the process. Based on these ongoing activities, a set of interim recommendations for further research are issued and briefly summarized in this paper.

\section{DELPHI SURVEY}

\subsection{Scope}

The Delphi method is one of the most well known approaches to systematically collect and analyze experts' opinions when forecasting the future [1], [2]. THINKcreative uses an adaptation of Delphi, combining the permanent core experts of the consortium with a large panel of experts gathered from different regions in Europe, as one of the instruments to identify major trends and likely future scenarios in collaborative forms. The panel of respondents was formed by 135 experts from industry (69\%) and academia (31\%), from the following countries: Portugal, Germany, The Netherlands, UK, Switzerland, Belgium, Czech Republic, Slovakia, France, Italy, and Sweden. A few participants from Mexico and USA were also involved in the survey.

As a starting basis, and through a number of brainstorming sessions, THINKcreative members identified 21 key drivers affecting the evolution of collaborative organizational forms (Fig. 1).

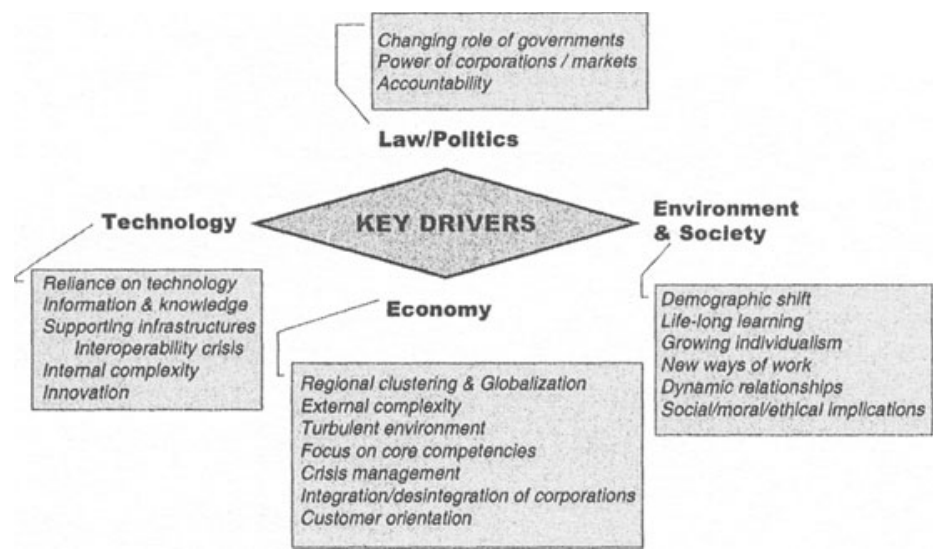

Figure 1 - Key drivers in collaborative organizations evolution

Out of this list, an extensive questionnaire of 67 questions was elaborated and the opinions of the panel experts with reference to a time frame of 5, 10,15 years, were collected. One example, for the "Innovation" driver, is shown in fig. 2. 


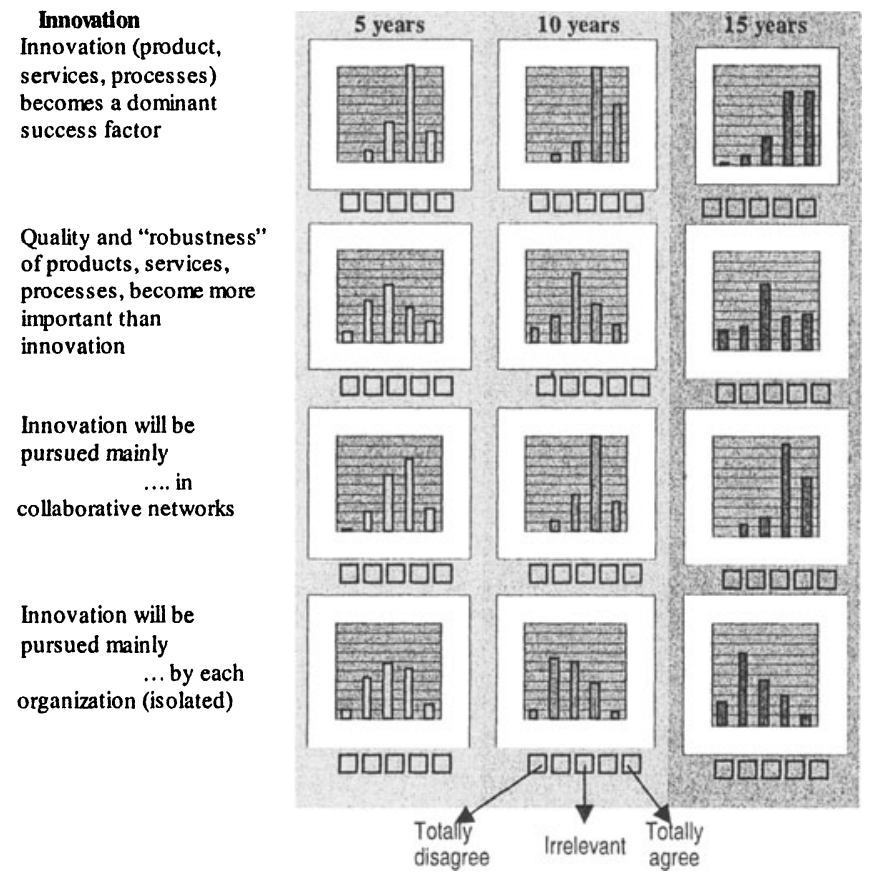

Figure 2 - Example of Delphi collected opinions

\subsection{Main findings}

The main conclusions of the Delphi survey can be summarized as:

\section{Regional clustering and Globalization}

- Although regional clusters, by reinforcing long-term relationships and leveraging local "culture", local specificities, and proximity to customers, might have in the short term some effect against the threats of globalization, the trends in the medium and long term are unclear.

\section{Customer orientation}

a Trends in product / services, especially in medium and long term, point to mass customization.

- Guaranteeing customer loyalty is clearly a determining competitive advantage.

- The responsibility of customer satisfaction is likely to become more diluted among network members, and no so much and exclusive responsibility of the customer interface node.

Internal complexity

口 Systems (e.g. manufacturing, service providers) will become increasingly complex, a trend that will become more clear in the medium and long term.

- There is a moderate tendency for products to become increasingly complex, namely in terms of their internal structure.

\section{External complexity}

B Business processes tend to be supported on highly dynamic and increasingly complex networks of collaborative entities, a trend particularly expected in the medium and long term, but not a so clear reality in the short term. 


\section{Turbulence environment}

a The speed of change in business environments is likely to increase, even in the short term, and a definitive expectation for the long term.

a The amount of change in business environments is likely to continue growing, as a moderate expectation for the short term and a clear trend in the long term.

Focus on core competences

a In order to remain lean and highly efficient in competing markets, organizations progressively trend to focus on their core competencies.

Changing role of governments

a Governments will not be an active part in the business environment, but will progressively play only a regulatory role.

\section{Power of corporations / markets}

a Corporations / markets will increase power, reducing the power of governments and smaller organizations.

- The role of networks of small, agile organizations as a reaction to this scenario is unclear.

Integration vs. disintegration of corporations

a The structure of large organizations is likely to evolve, in the medium and long term, namely in the form of organized disintegration (forming complex networks of interacting units).

Reliance on technology

- Modern economy and society in general will increasingly rely on technology.

- In the medium and long term there will be a progressive emergence of a posttechnology era with more emphasis put on the human skills.

\section{Accountability}

ㅁ There will be a growing accountability on ethical, environment, financial, and social issues.

Although already a trend, this scenario will become clearer in the medium and long term.

\section{Information and knowledge}

There is a clear expectation that organizations will more and more struggle with information and knowledge overload.

- At the same time, decision-making processes will be more based on a sound information and knowledge basis.

\section{Demographic shift}

a It is likely that the aging of population will accelerate the emergence of neworganizational forms in the medium and long term.

․ But the aging of population will be progressively compensated by increased migration flows, which in turn might lead to an increase of socio-cultural clashes.

Life-long learning

․ Life-long learning will become widely recognized and supported by organizations.

- In the medium and long term, as a reaction to the market demand, universities will change their teaching models in order to offer life-long learning programs.

at the same time new curricula have to be defined by universities and training institutions, including new competencies that are fundamental to assure new organizational forms and ways of doing business. 


\section{Growing individualism}

․ There is an expectation that in the medium term and especially in the long term collaborative behavior and strong human networks (professional and social) will grow as a reaction to a competitive fast changing society.

a In parallel, also in the medium and long term, it is expectable that new social systems will emerge to provide for new contractual relationships between individuals and organizations.

In the short term, under increasing competition and social turbulence, the social trends are unclear.

New ways of work

口 In the short and medium term, new ways of work (e.g. tele-work, e-freelancing, entrepreneurships) will stay confined to specific sectors.

․ In the long term there is a moderate expectation that new ways of work will become more significant $(20-55 \%)$.

Dynamic relationships

- In the medium and long term we might observe a growth in dynamic shortterm collaborative relationships, especially within limited contexts (e.g. regional clusters).

- In the short terms it remains unclear whether the collaborative relationships will be established mainly on a long-term basis or on a short-term dynamic basis.

\section{Innovation}

$\square$ Innovation on products, services, and processes will clearly become a dominant success factor.

Innovation will be pursued mainly in collaborative networks, especially in the medium and long term.

\section{Social / moral / ethical implications of virtual collaborative environments}

a It is not expectable that society will react against virtual collaborative environments (i.e. a push-back in technological development is not foreseen). On the contrary the society will progressively learn to make the best use of virtual collaborative environments and technology.

\section{Crisis management}

a There is a moderate expectation that, in the medium and long term, crisis management will become a standard "capability" in organizations.

- At the same time, crisis management might become a specialized activity (market niche) for some specific entities.

\section{Support infrastructures}

In the short term current technologies could provide adequate support for agile I dynamic cooperative organizations but the lack of common infrastructures and business support functions is regarded as an obstacle.

- In the medium and long term the trends are unclear, which might be related to the uncertainty regarding the requirements posed by new organizational forms.

Interoperability crisis

In the short and medium terms, the lack of interoperability of SW/HW systems represents a crucial obstacle in developing new forms of cooperation. This obstacle is however attenuated by engineering efforts.

In the long term, the interoperability scenario is unclear. 


\section{THEMATIC AND REGIONAL WORKSHOPS}

\subsection{General}

A second mechanism to identify trends in organizational forms and related research needs was the organization of a series of workshops and discussion panels. These events were organized as consortium meetings (5) involving invited external experts, panels associated to international conferences (2), or as regional workshops (10) involving local industry and academic visionaries. In particular through the regional workshops, it was possible to involve more than 400 participants that otherwise would not travel abroad to attend any event in a major European city.

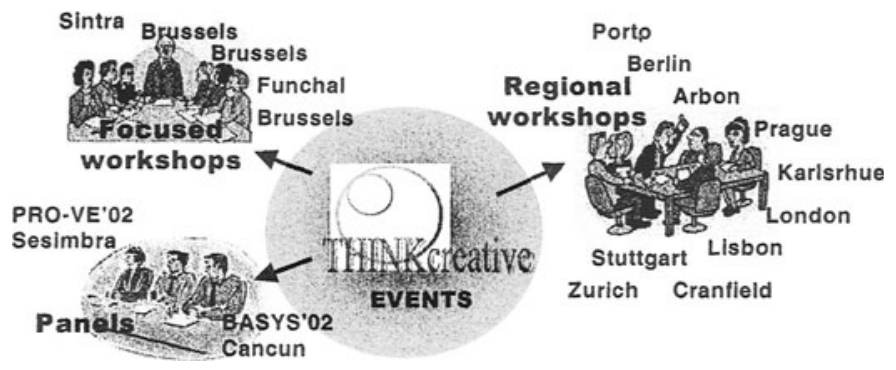

Figure 3 - THINKcreative events

The regional workshops were devoted to the topic "Towards collaborative relationships in the Creative Economy" and discussions were guided by three main questions:

1. Which inter-organization collaborative relationships will emerge (new forms) or will be further developed (current forms) within the next 5, 10, 15 years?

2. Which IT tools and functionalities have to be developed / improved in order to support those collaborative relationships?

3. Which socio-organizational, legal and ethical frameworks will result / or are needed to support the new collaborative relationships?

\subsection{Main recommendations}

The main integrated conclusions of the various workshops and panels can be summarized in the following recommendations:

- Socio-economic and organizational challenges. Considerable more effort has to be spent on fundamental theoretical work, namely in the following areas: Fundamental conceptual organizational research, management of business innovation, generic organizational and business models, migration and change towards VO, organization, development, and management of project teams, semantic-informed self-organizing structures, and collaborative knowledge networks, and virtual communities / communities of practice.

- Ethical and moral issues. Ethical and moral issues confront the traditional management in the most fundamental aspects of building and operating any 
business. A sustainable new economy needs to be based on sound foundations and any advanced research program on collaborative networks shall give these traversal issues proper attention, starting with three basic questions: Where do our ethics and moral come from? How can VOs respond to the ethical and moral questions? What are the initial ethical and moral questions facing VOs?

- Formal models and theoretical foundations. One of the main weaknesses in the area, which prevents a proper understanding and also its recognition as a scientific discipline, is the lack of appropriate theories, consistent paradigms and formal modeling tools, in order to concisely describe it. It is therefore urgent to launch fundamental research on theoretical foundations and identification of suitable formal modeling approaches that better facilitate the definition / characterization of collaborative organizations. Examples of topics of interest here include: formal theories, graph theory, normative models, visual modeling, theories of complexity, algebraic reference models, ontologies, multi-agent systems, game theory, modeling social aspects of collaborative networks such as social actors networks, soft modeling, simulation and experimental modeling, and models integration / models interoperability.

I ICT infrastructures. There is a prominent need to further research towards the establishment of generic, interoperable, pervasive, free (low-cost) and invisible (user-friendly) infrastructures. Key issues include: identification, design, and development of required base reference architectures and innovative approaches to advanced federated / distributed information management, interoperability mechanisms and tools, distributed activity coordination and languages, and integration of legacy systems, inter-domain transactions, recovery mechanisms, tracking and auditing services, among others.

- Breeding environment management and VO creation. Important challenges include: Management of VO breeding environments (e.g. regional clusters), VO creation frameworks, trust and regulation of electronic support institutions (e.g. e-notary and certification services), modeling and management of contracts and cooperation agreements, negotiation, methodologies for transforming existing organizations into VO-ready organizations, etc.

Collaborative networks operation support. Key areas requiring urgent attention include: Coordination, administration and management of highly distributed activities, risk management and assessment tools, development of value addedservices, dynamic evaluation of revenues, rights and liabilities, in combination with the understanding of new value systems, soft-modeling and reasoning, econtract management, advanced simulation tools for collaborative networks, and new user interfaces seeking an entertainment facet as a way to overcome cultural barriers.

- Support VO evolution and dissolution. Examples of key research challenges include: Definition of legal and organizational frameworks for terminating cooperation processes, mechanisms for handling post-cooperation IPRs and liabilities, VO inheritance management, and traceability mechanisms.

- Virtual Laboratories (VL) and remote supervision. In addition to the generic infrastructure needs, further issues include: Coordinated and dynamic resource sharing for collaborative problem solving, high-performance data integration, tele-supervision and tele-operation assisted by intelligent mobile agents, 
facilities for definition of information and services access rights and visibility levels, representation languages for cooperation formalization, social aspects of remote collaboration, support for asynchronous cooperation and delegation, extended error recovery, training methodologies based on VL for manufacturing professionals, new integrated and comprehensive methodologies for shop-floor reengineering, combination of re-engineering and remote supervision approaches.

- Professional Virtual Communities (PVC). Require additional aspects such as: Development and management of shared, smart spaces for geographically distributed teams, understanding and modeling multi-level relationships in contract-bound communities, coordination and notification mechanisms, frameworks for collaboration in mobile contexts, and mechanisms to handle Intellectual Property in PVCs under social contracts.

\section{THEORETICAL FOUNDATION}

\subsection{Theories and modeling approaches}

One of the main weaknesses in the area of collaborative, networked organizations is the lack of appropriate theories, consistent paradigms and formal modeling tools. Dramatically enough there is not yet a common definition of basic concepts such as virtual organization or virtual enterprise. This situation constitutes a major obstacle for interaction among experts from the multiple disciplines that should play a role in the area and also an obstacle for the recognition of virtual organizations as a scientific discipline.

During last decades research in Europe (and partially in the USA) has become too pragmatic, focused on short-term results, without enough attention to the fundamentals. To aggravate the situation most engineers nowadays ignore what a "formal theory" is.

Nevertheless, some theories and paradigms defined elsewhere have been suggested by several research groups as promising tools to help understand and characterize emerging collaborative organizational forms. A number of workshops organized in the framework of the THINKcreative initiative, involving a large number of European and American experts from different disciplines, helped in reaching a first identification and characterization of promising approaches (Table 1).

Table 1: Examples of promising approaches

\begin{tabular}{|c|c|c|}
\hline Princlples & Applicability & Limitations / Challenges \\
\hline \multicolumn{3}{|l|}{ Formal languages } \\
\hline $\begin{array}{l}\text { Rigorous (algebraic) } \\
\text { Abstract declarative } \\
\text { logic } \\
\text { Systematic design }\end{array}$ & $\begin{array}{l}\text { Model structure and operational } \\
\text { behavior } \\
\text { Formulate and verify operational } \\
\text { plans } \\
\text { Validate conformity between } \\
\text { specifications and implementation }\end{array}$ & $\begin{array}{l}\text { Difficult to develop and } \\
\text { understand } \\
\text { Need practical application } \\
\text { methodology } \\
\text { Need methods to bridge the gap } \\
\text { between abstract formalism and } \\
\text { IT implementation }\end{array}$ \\
\hline \multicolumn{3}{|l|}{ Graph theory } \\
\hline Sound math foundation & Represent networks of relationships & Basic theory is very rigid - needs \\
\hline
\end{tabular}




\begin{tabular}{|c|c|c|}
\hline $\begin{array}{l}\text { Graphical represent. of } \\
\text { entities \& relationships } \\
\text { Support computation }\end{array}$ & $\begin{array}{l}\text { - topology, routing, activity, flow } \\
\text { Perform computations on flows } \\
\text { Optimization }\end{array}$ & $\begin{array}{l}\text { extensions to represent non } \\
\text { tangible, qualitative relationships } \\
\text { (fuzzy dimensions), and multi- } \\
\text { criteria }\end{array}$ \\
\hline \multicolumn{3}{|l|}{ Complexity theory } \\
\hline $\begin{array}{l}\text { Non-linear mathematics } \\
\text { Dynamic systems }\end{array}$ & $\begin{array}{l}\text { Analysis of self-organizing behavior } \\
\text { Learn how to manage chaotic } \\
\text { dynamics } \\
\text { Insights on VO behavior ("small- } \\
\text { worlds") }\end{array}$ & $\begin{array}{l}\text { Models from other domains (e.g. } \\
\text { biology, physics) are difficult to } \\
\text { apply to VOs } \\
\text { Need further work on complex } \\
\text { systems with social actors } \\
\text { (humans) }\end{array}$ \\
\hline \multicolumn{3}{|l|}{ Multi-agent systems } \\
\hline $\begin{array}{l}\text { Agent concept } \\
\text { Agency } \\
\text { Distributed AI }\end{array}$ & $\begin{array}{l}\text { Model societies of autonomous, } \\
\text { heterogeneous, evolving entities } \\
\text { Coalition formation and negotiation } \\
\text { Simulate self-organizing behavior }\end{array}$ & $\begin{array}{l}\text { Need further developments in } \\
\text { social aspects of agency, dealing } \\
\text { with uncertainty and } \\
\text { interoperation with other models } \\
\text { (e.g. process modeling languages) }\end{array}$ \\
\hline \multicolumn{3}{|c|}{ Semiotics and deontic logic } \\
\hline $\begin{array}{l}\text { Semiotics \& cognitive } \\
\text { theory } \\
\text { Logic of obligations } \\
\text { Normative approach }\end{array}$ & $\begin{array}{l}\text { Model responsibility relationships } \\
\text { and commitments } \\
\text { Prescribe norms and roles, legal } \\
\text { support } \\
\text { Capture system requirements }\end{array}$ & $\begin{array}{l}\text { Reasoning in deontic logic may } \\
\text { lead to paradoxes; difficult to } \\
\text { automate reasoning } \\
\text { An promising approach: } \\
\text { integration with agent logic }\end{array}$ \\
\hline \multicolumn{3}{|l|}{ Game theory } \\
\hline $\begin{array}{l}\text { Collaborative games } \\
\text { Decision theory } \\
\text { Probabilities }\end{array}$ & $\begin{array}{l}\text { Model coalition formation and } \\
\text { support conflict resolution } \\
\text { Distribution of resources } \\
\text { \&responsibility }\end{array}$ & $\begin{array}{l}\text { Weak capture of subjective } \\
\text { relationships } \\
\text { Need better integration with } \\
\text { network analysis methods } \\
\text { Need to extend dynamic } \\
\text { capabilities }\end{array}$ \\
\hline \multicolumn{3}{|l|}{ Social actors networks } \\
\hline $\begin{array}{l}\text { Extension of graph } \\
\text { theory to include } \\
\text { relationships between } \\
\text { social actors }\end{array}$ & $\begin{array}{l}\text { Model and analyze social and } \\
\text { organizational structure of } \\
\text { collaborative networks - elements of } \\
\text { sociability, power structures, etc. }\end{array}$ & $\begin{array}{l}\text { Still in its early phase } \\
\text { Covers only the structural view, } \\
\text { no agency } \\
\text { Need to be integrated with BPR } \\
\text { method } \\
\text { Need to extend fuzzy dimensions }\end{array}$ \\
\hline \multicolumn{3}{|c|}{ Temporal and modal logics } \\
\hline $\begin{array}{l}\text { Abstract declarative } \\
\text { logic }\end{array}$ & $\begin{array}{l}\text { Model the operational phase aspects } \\
\text { Synthesis of processes }\end{array}$ & $\begin{array}{l}\text { Complex, as any formal method } \\
\text { Need to integrate structure } \\
\text { descriptions }\end{array}$ \\
\hline \multicolumn{3}{|l|}{ Metaphors } \\
\hline $\begin{array}{l}\text { Informal tools for } \\
\text { human communication } \\
\text { (mostly graphic with } \\
\text { super-imposed text, } \\
\text { wisdom sentences, etc) }\end{array}$ & $\begin{array}{l}\text { Quick description for human } \\
\text { communication (a possible help in } \\
\text { expressing complex ill-defined } \\
\text { concepts) } \\
\text { Use in early stages (conceptual } \\
\text { design) }\end{array}$ & $\begin{array}{l}\text { Risk of taking metaphors too } \\
\text { strictly } \\
\text { Need further evaluation and } \\
\text { research in consistent } \\
\text { understanding in the creation and } \\
\text { interpretation of metaphors } \\
\text { Need to combine with formal } \\
\text { methods }\end{array}$ \\
\hline
\end{tabular}

Nevertheless, there is no single formal modeling tool / approach that adequately covers all perspectives - no "universal language" for all problems. For instance, network dynamics represents a multi-dimensional problem that requires qualitative and quantitative modeling and reasoning approaches. Furthermore, there are a large number of different perspectives in collaborative, networked organizations, which can be modeled from multiple perspectives or for different purposes. Even for each 
modeling perspective there might be a need to use more than one tool. Therefore in the recommended approach interoperability of different modeling tools and approaches is considered a must for a comprehensive definition of this organizational paradigm.

\subsection{Emerging behavior}

The networking opportunities offered by new technologies are good catalyzers for new and continuously evolving organizational forms and emergent behaviors. Understanding the emergent behaviors is thus a major challenge that requires further fundamental research. In this direction, and by combining contributions from different disciplines, including complex systems theory, biology, artificial intelligent I multi-agent systems, game theory, chaos theory, etc., another THINKcreative workshop was organized to identify and characterize research challenges, promising approaches, and major goals towards the development of new models and technology support for the prediction and management of emerging behavior in future dynamic collaborative networks. As a result, the following recommendations are made:

a Understanding and modeling emerging behavior in new collaborative, networked organizations is a key research challenge and a pre-requisite for the design and development of new support infrastructures. The concept of "emergence" itself and the balance of emergent vs. deliberative strategies need to be better understood in this context.

- Future research programs in advanced virtual organizations and new collaborative networked organizations must effectively combine and integrated contributions of multiple disciplines, namely Computer Science and Engineering, Economy, Sociology and Business Policy and Strategy, Organizational and Management Sciences, receiving inputs from other fields such as Biology, Ecology and Physics, but also taking into account Moral and Ethical issues. Interesting approaches to analyze evolution of networks are coming, for instance, from Physics [6].

- The various theories of complexity can give some insights on how to approach the analysis of new organizational forms and their emerging behavior. It is clear that we cannot simply adopt models developed in other contexts (e.g. Physics and Biology) as the "human-social" elements are difficult to model, but the methodologies and basic principles researched in the area of complexity represent a promising starting point.

- The multi-agent community has been addressing many problems similar to the ones found in VOs. In fact many aspects of the VOs and their behavior, namely coalition formation and emergent behavior, can be modeled by the MAS concepts and Distributed AI. A closer interaction between the two areas is recommended.

- In particular multi-agent approaches can bring important inputs to the coalition formation processes, but more work is needed in terms of the social aspects of agency, normative models and reasoning with uncertainty.

- The concept of meta-agent represents a promising approach to help identifying 
trends and patterns in the behavior of networked organizations.

a There are significant standardization efforts in MAS, namely in the framework of FIPA, but the specific needs of VO haven't been addressed yet - an open opportunity for cooperation between the two communities.

- Knowledge-based approaches are certainly required but the emphasis has to be put on soft modeling (uncertain / imprecise knowledge), multi-level modeling and on capturing the socio-cultural dynamics.

- A multi-worlds / multi-level perspective shall be considered when modeling and designing support infrastructures (Social / Cultural, Legal / Explicit, Physical).

- Simulation and experimental modeling approaches, methodologies and tools specifically designed for networks need to be developed as a means to help grasping the emerging shapes and behavior of unpredictable evolutionary systems.

- It is necessary that the issues of advanced VOs to be addressed following a holistic approach instead of trying to solve one problem at a time; the traditional fragmented approach tends to create new problems whenever partial solutions are put forward.

a Considering that not a single formalism / theory is enough to represent / capture all aspects of emerging organizational forms and behavior, there is a need to explore theories and formalisms from other areas and put them in the appropriate context. The area definitely requires more formal and sound theoretical background support. Nevertheless there is also the need to consider understandability of models / theories in order to have them accepted by industry (namely SMEs).

a There is a need to invest on a better understanding of underlying mechanisms of cooperation and competition and the underlying principles of self-organizing systems. Work on learning webs can give some insights in this direction.

- Understanding what is turbulence, how to identify opportunities, what are the breeding conditions for innovation, are important areas requiring more theoretical and experimental work.

- More theoretical work is also needed on understanding the roles of leadership (and what kind) and coordination, the scope of vision/mission in organizations, the emergence and establishment of coordination rules and "normative" institutions.

$\square$ There is a need for a new ethical approach in the establishment of networked organizations as the traditional economic model is no longer appropriate; current approaches to issues such as anti-trust/unfair competition must be revised.

\section{CONCLUSIONS}

Foreseeing the future in a rapidly evolving environment is certainly a hard if not impossible task. In fact it became evident during the various events organized by 
THINKcreative that experts and industry visionaries more easily identify needs and even obstacles than they give an opinion about future trends and plausible scenarios, specially for the short term.

It was therefore important to collect a substantial number of experts' opinions from different sectors, but it shall be noticed that the purpose of this study is not to reach a majority consensus through a simple statistical process. More important than reaching a sort of "statistical consensus" is to collect and integrate input from carefully selected visionaries and industry leaders from different domains and disciplines. It is also important to note that more important than a forecasting exercise, what really matters is to build a "desirable vision" of the future, as well as pointing the way how to progress in that direction. In this perspective, the focus moves from "passive guessing"(like in traditional scenario planning works) to "active planning". It is however certain that "planning the future" needs to consider the trends and plausible cases and scenarios that will constrain the plan and even the vision.

Finally it is important to note that unlike the USA, Europe lacks a tradition (and skilled people) for planning the future. The EC research policies of the last years appear to have created a "culture of the short term" in the community. Namely, the tremendous pressure for achieving immediate results is acting as a major barrier to real innovative breakthroughs.

The list of recommendations from THINKcreative is a contribution to the establishment of a new research agenda on collaborative networks aiming at a real breakthrough towards a major impact in science and society. This paper summarizes only the preliminary results of the THINKcreative initiative. The final results and recommendations will be published in the form of a Green Report [5].

Acknowledgements. The work described in this paper was funded in part by the European Commission through the IST project THINKcreative. The author thanks the collaboration and contributions of all consortium partners.

\section{REFERENCES}

1. Committee on Visionary Manufacturing Challenges - Visionary Manufacturing Challenges for 2020, National Academy Press, Washington, 1998.

2. H. A. Linstone, M. Turoff - The Delphi method: Techniques and Applications, New Jersey Institute of Technology, 2002, www.is.njit.edu/pubs/delphibook/index.html.

3. L. M. Camarinha-Matos, H. Afsarmanesh - THINKcreative Interim Report on New Collaborative Forms and Their Needs, Sept 2002. www.thinkcreative.org.

4. L. M. Camarinha-Matos, H. Afsarmanesh, "Dynamic Virtual Organizations, or not so dynamic?", in Proceedings of BASYS'O2 - Knowledge and technology integration in production and services, Kluwer Academic Publishers, ISBN 1-40207211-2, pp. 111-124, Sept 2002.

5. L. M. Camarinha-Matos, H. Afsarmanesh (ed.s) - Collaborative Networked Organizations, to appear, Kluwer Academic Publishers, 2003.

6. S. N. Dorogovtsev, J. F. F. Mendes - Evolution of networks - From biological nets to the Internet and WWW, Oxford University Press, ISBN 0-19-851590-1, 2003. 